O Open Access Full Text Article

\title{
A retrospective six-patient series of apatinib for the treatment of persistent or recurrent carcinoma of the cervix
}

This article was published in the following Dove Press journal: OncoTargets and Therapy

\section{Chao Chen \\ Shukui Qin \\ Zixiong Li \\ Xianwen Luo \\ Yu Zhang \\ Jue Zhang \\ Xiufeng Liu}

Department of Medical Oncology of PLA Cancer Center, Jinling Hospital, Nanjing 210002, People's Republic of China
Correspondence: Xiufeng Liu Department of Medical Oncology of PLA Cancer Center, Jinling Hospital, No 34, 34 Biao Yanggongjing Street, Qinhuai

District, Nanjing 210002, People's Republic of China

Email njjloncologylxf@I63.com
Objective: Although advances have been made in the clinical and therapeutic management of women with cervical cancer, the best treatment for patients with metastatic or recurrent cervical cancer is still undefined. Apatinib, a novel inhibitor of vascular endothelial growth factor receptor-2 tyrosine kinases, has been successful in treating various malignancies. This study was conducted to evaluate the efficacy and safety of apatinib in the treatment of recurrent cervical cancer.

Methods: Patients with recurrent cervical cancer received apatinib after failure of the second- or higher-line chemotherapy. Apatinib was administered as $500 \mathrm{mg}$ daily on days 1 through 21 of each 4 -week cycle. The primary endpoint was overall survival (OS), and the secondary endpoints included progression-free survival (PFS), objective response rate (ORR), disease control rate (DCR), and treatment-related adverse events were reviewed and evaluated.

Results: Six patients were administered apatinib for at least one complete cycle. The median OS was 16.0 months (95\% CI: 6.8-25.2), and the median PFS was 7.0 months (95\% CI: 2.2 11.8), One patient achieved partial response and three patients achieved stable disease. Two patients were evaluated as progression disease. The ORR was $16.7 \%(1 / 6)$ and the DCR was $67.7 \%(4 / 6)$. The common side effect of apatinib was hypertension; however, the toxicity of apatinib was tolerable and controllable.

Conclusions: Apatinib is an option in the treatment of recurrent cervical cancer after failure of the second- or higher-line chemotherapy. Further prospective evaluation of the utility of apatinib is required.

Keywords: cervical cancer, apatinib, efficacy, side effects, angiogenesis

\section{Introduction}

Despite the significant progress in the last half century, cervical cancer is still the fourth most common cancer affecting women worldwide. ${ }^{1}$ The morbidity and mortality have been reduced in the developed country due to the cytological screening program, the identification of human papillomavirus (HPV) as the key etiological factor, and HPV vaccination. However, in the low-resource region where it is difficult to access the screening program, cervical cancer is the second most common malignancy in women, with over $85 \%$ of the lethal cases caused by cervical cancer. ${ }^{2}$ Early stage and localized cancers maybe cured by surgery, chemoradiotherapy, or both, but advanced and recurrent cases are often incurable. For the majority of patients with advanced/recurrent malignancy, palliative chemotherapy is the optimized treatment. Unfortunately, short-lived response to chemotherapy 
in patients with advanced cervical cancer indicates that this malignancy is relatively chemo-resistant. Most responses to chemotherapy are partial and short in duration, therefore, and more effective treatment for advanced/ recurrent cases after standard first-line chemotherapy has yet to be developed.

Angiogenesis, blood vessel formation, and remodeling are a central progress for the cancerous generation and development. $^{3}$ Vascular endothelial growth factors (VEGFs) and their receptors (VEGFRs) mediate the tumor angiogenesis in cervical cancer. ${ }^{4}$ There are several subtypes are both VEGF family and VEGFR family which has been reviewed in detail. VEGFA and its receptors VEGFR2 play major roles in angiogenesis. ${ }^{5}$ In terms of carcinogenesis in cervical cancer, HPV mediates vascular formation via the VEGF-VEGFR signaling pathway. Overexpression of HPV oncoprotein enhanced VEGFA expression which consequently promotes vascular generation. It is of note that vascular seen at colposcopy are hall marks for invasive stage. ${ }^{6}$ Pathologically, increased VEGFR2 expression and microvessel density are correlated with metastasis and poor prognosis, making anti-vascular therapy to be a promising approach to the treatment of invasive cervical cancer. ${ }^{7}$

Since clinical trial GOG227, numerous studies highlight feasibility and importance of the anti-vascular therapy in cervical cancer. ${ }^{8}$ In these studies, although the bevacizumab has been approved as the first-line strategy for advanced/recurrent cervical cancer in developed countries, some factors, such as its cost, hamper its broad application especially in low-resources region where most refractory cases occur. ${ }^{9}$ Affordable and safe agents are unmet for these patients. In light of anti-angiogenesis therapy, blocking VEGFR, the receptor of VEGF, might be an alternative way for the blocking newly vascular generation. As a novel VEGFR2 inhibitor, apatinib could strongly inhibit new vascular formation by blocking tyrosine kinase (TKI) activity. Phase I and II clinical trials have shown the powerful anti-tumor activity and manageable side effects in various cancers such as non-small-cell lung cancer, mammary cancer, and gastric cancer. ${ }^{10}$ Of importance, apatinib is currently also being studied for its anti-cancer efficacy in cervical cancer, with a recent study indicating promising effects both in vitro and in vivo. ${ }^{11}$ However, the report about the affection and safety of apatinib in cervical cancer is not available to our knowledge, although it could be an option for heavily treated patients.
As a consequence of these considerations, we accessed the response and safety of apatinib in patients suffering from heavily pretreated advanced/recurrent cervical cancer. These patients have accepted the standard procedure of anti-cancer treatment, however showed failure after first-line chemotherapy. In addition to the results of the retrospective analysis of apatinib usage in these patients, an additional literature review of TKI in cervical cancer was performed.

\section{Patients, materials, and methods Patients}

Patients with recurrent cervical cancer received apatinib after failure of the second- or higher-line chemotherapy at the Department of Medical Oncology of PLA Cancer Center, Jinling hospital from August 2016 to January 2019 were included in this retrospective analysis. Treatment failure was defined as disease progression or intolerable adverse effects during the treatment period with chemotherapy. This study was conducted in accordance with the principles of the Declaration of Helsinki and was formally approved by the ethics committee of Jinling hospital (2016). Prior to apatinib therapy, all patients had been given verbal explanations and had signed informed consent forms.

\section{Study design}

Baseline patient evaluation included a history and standardized physical examination, routine laboratory screening, CT scanning, bone marrow evaluation, electrocardiogram, and cardiac ultrasonography. At least one site of disease had to be measurable in these patients. Clinical pathological data at initial diagnosis and last recurrence were accessed.

\section{Treatment}

Apatinib was administered daily on days 1 through 21 of each 4-week cycle. Apatinib $500 \mathrm{mg}$ every day was selected as the standard dose. In an intolerable side effect, the dose was reduced to $375 \mathrm{mg}$ or $250 \mathrm{mg}$ every day. Treatment interruption up to 7 days was allowed if severe toxicities developed.

\section{Data assessments}

Tumor measurements were carried out after two, four, and six treatment cycles. Thereafter, this was done every three months until disease progression or dead. Complete 
response (CR) means all target lesions completely disappeared lasting for at least four weeks. Partial response (PR) was defined as the tumor was reduced $\geq 30 \%$ with no new malignant lesion for at least four weeks. Stable disease (SD) was defined as the sum of the maximum diameter of the target lesion reduced to less than PR, or increased to less than progression disease (PD). PD was defined as $\mathrm{a} \geq 20 \%$ increase in the maximum diameter of the target lesion, or new malignant lesions occur. Disease control rate $(\mathrm{DCR})=(\mathrm{CR}+\mathrm{PR}+\mathrm{SD}) /$ total cases, and the objective response rate $(\mathrm{ORR})=(\mathrm{CR}+\mathrm{PR}) /$ total cases. The PFS was calculated from the first date of enrollment to disease progression, relapse, or death from any cause, or the last date of follow-up. The OS was measured from the date of enrollment to the date of death for any reason or to the last date of follow-up. The National Cancer Institute Common Terminology Criteria for Adverse Events version 4.03 was used to categorize and grade the adverse events (AEs).

\section{Statistical analysis}

OS, PFS, and their 95\% Confidence Intervals (95\% CI) were estimated by the Kaplan-Meier method and compared with the log-rank test. All the statistical analyses were performed using SPSS software, version 21.0 (SPSS Inc., Chicago, IL, USA).

\section{Results}

\section{Patient characteristics and treatment}

From August 2016 to January 2019, six patients with a median age of 54.5 years (range 41-66 years) received apatinib treatment (Table 1). All patients had an International Federation of Gynecology and Obstetrics (FIGO) Stage IV disease. Five patients $(5 / 6,83.3 \%)$ had received radiotherapy, and three patients (3/6, 50.0\%) received prior wide resection. All patients received prior chemotherapy as outlined in the National Comprehensive
Cancer Network clinical practice guideline. Six patients were administered apatinib for at least one complete cycle, and then the survival analysis as well as security data are listed in Table 2. No patients developed intolerable symptoms of toxicity or withdrew consent from the study.

\section{Efficacy}

Six patients were evaluated for the declared study efficacy endpoints (Table 2). One patient achieved PR. Three patients achieved SD. Two patients were evaluated as PD. The ORR was $16.7 \%$ (1/6) and the DCR was $67.7 \%$ $(4 / 6)$. Tumor shrinkage was noted in three (3/6) patients (Figure 1). The median overall survival (OS) was 16.0 months (95\% CI: 6.8-25.2), and the median progressionfree survival (PFS) was 7.0 months (95\% CI: 2.2-11.8) (Figure 3).

The imaging and staging data of a typical case were shown as follows. One 36-year-age patient was initially diagnosed to have IIb stage cervical cancer in August 2012. She was treated by radical chemoradiotherapy. In 2015, she was found recurrent in the lymph node. After six cycles of chemotherapy including paclitaxel and cisplatin, she was evaluated as PD. Then, apatinib was administered as $500 \mathrm{mg}$ daily. At the first tumor measurement, CT scanning showed a significant reduction of metastatic lesions and this patient was considered to be PR (Figure 2). She is continuously taking apatinib.

\section{Toxicity}

Treatment-related AEs were assessed throughout the study (Table 3). A few patients experienced drug-related reaction, most of these adverse reactions were mild and tolerable. The most common adverse drug reactions were hypertension (4/6), albuminuria (2/6), mucositis $(2 / 6)$, anorexia (1/6), fatigue $(2 / 6)$, mucositis $(2 / 6)$, thrombocytopenia (1/6), anemia (1/6), leukopenia $(1 / 6)$, and handfoot syndrome (1/6) (Table 3 ). Major grade 3 adverse drug

Table I Patients characteristics

\begin{tabular}{|c|c|c|c|c|c|c|c|c|}
\hline $\begin{array}{l}\text { Patient } \\
\text { no }\end{array}$ & $\begin{array}{l}\text { Age at } \\
\text { diagnosis }\end{array}$ & $\begin{array}{l}\text { Tumor stage at } \\
\text { diagnosis }\end{array}$ & Differentiation & Surgery & Radiotherapy & Chemotherapy & $\begin{array}{l}\text { Course of } \\
\text { disease }\end{array}$ & $\begin{array}{l}\text { OS } \\
(\mathrm{m})\end{array}$ \\
\hline I & 36 & Ilb & Squamous G2 & No & Yes & Yes & PR & 21 \\
\hline 2 & 56 & Ila & Squamous G3 & Yes & Yes & Yes & $S D$ & 7 \\
\hline 3 & 46 & $\mathrm{IVb}$ & Squamous G3 & Yes & Yes & Yes & $S D$ & 12 \\
\hline 4 & 53 & llb & Squamous G2 & No & Yes & Yes & $S D$ & 16 \\
\hline 5 & 51 & Ila & Squamous G2 & No & No & Yes & PD & 10 \\
\hline 6 & 53 & Illb & Squamous G3 & Yes & Yes & Yes & PD & 6 \\
\hline
\end{tabular}


Table 2 Baseline of patients' characteristics

\begin{tabular}{|l|l|}
\hline Characteristic & $\mathbf{N}=6$ \\
\hline Age (years) & \\
\hline $\begin{array}{l}\text { Median } \\
\text { Range }\end{array}$ & $\begin{array}{l}49.2 \\
36-56\end{array}$ \\
\hline ECOG & \\
\hline $0-1$ & 5 \\
2 & 1 \\
\hline Prior therapy & \\
\hline $\begin{array}{l}\text { Operation } \\
\text { Radiotherapy } \\
\text { Chemotherapy }\end{array}$ & 3 \\
\hline
\end{tabular}

Waterfall plot for change in tumor burden

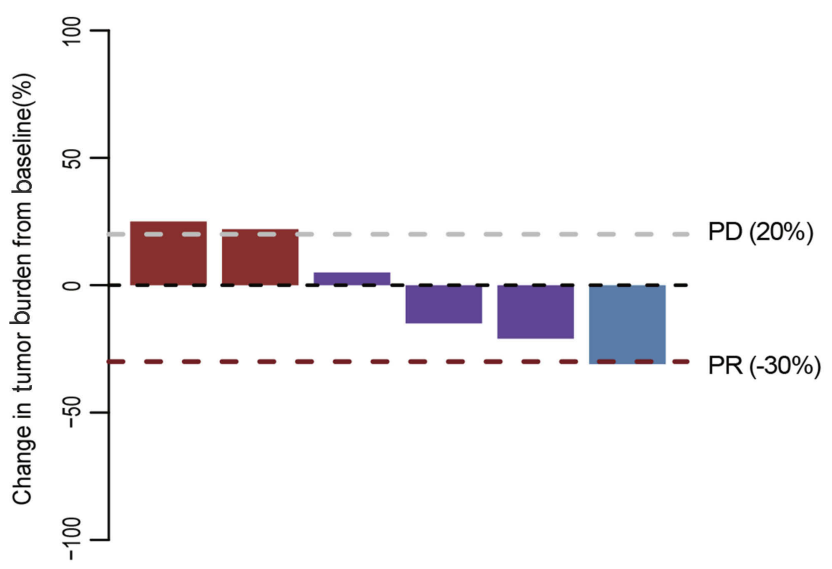

Figure I Waterfall plot for the best percentage change in target lesion size. Abbreviations: PD, progression disease; PR, partial response.

reactions were hypertension (1/6), albuminuria (1/6), thrombocytopenia (1/6), and anemia (1/6). No grade 4 AE was observed in the study. Three of these cases were treated with dose-modulated apatinib therapy for nonhematologic toxicities. Hand-foot syndrome and proteinuria could be relieved by dose reduction. Therefore, AE monitoring and dosage adjustment are effective methods for reducing the drug toxicity. No treatment-related severe AE was observed in our study.

\section{Literature reviewing}

In total, there are five reports about the application of TKI in cervical cancer. These data are summarized in Table $4 .^{12-16}$

\section{Discussion}

Novel strategies, based on our understanding of biology and genetics, are desperately needed to treat common malignancies such as cervical cancer that are not sensitive to current cytotoxic chemotherapy. Anti-angiogenesis is a well-established strategy in this setting, and apatinib is an oral angiogenesis inhibitor specifically targeting VEGFR2 via blocking the activity of TKI. This novel agent is well tolerant and highly active in some solid cancers and was recently found effective against cervical cancer in vitro. Apatinib was administered orally within clinical routine practice because of the lack of an established choice after second-line options. After this treatment, we found an ORR of $66.7 \%$, a median PFS of 7.0 months, and a median OS of 16.0 months. To our knowledge, the current study is the first to describe the clinic use of apatinib in patients suffering advanced/recurrent cervical cancer.

In addition, to block the VEGF signaling pathway by working as TKI inhibitor, apatinib also inhibits the ABC (ATP-binding cassette) proteins which play critical rules in chemo-resistance. ${ }^{17}$ It is well understood that $\mathrm{ABC}$ protein protects cancer cells by pumping out toxicity drug. In the preclinical study, concomitant treatment with paclitaxel, a most used toxicity drug, apatinib significantly increased the susceptibility to paclitaxel in either cervical cancer cells or the mouse model. ${ }^{11}$ Cisplatin is the most effective single chemoreagent for cervical cancer. Unfortunately, cisplatin treatment frequently results in the development of drug resistance after initial responses in various cancer. ${ }^{18}$ Apatinib can restore the sensitivity of cancer cells to cisplatin by down-regulating the expression level of multi-drug resistance- 1 and inhibiting the

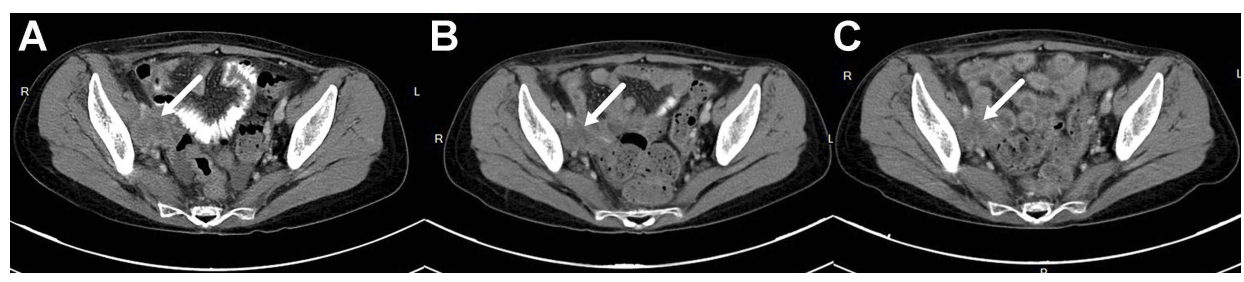

Figure 2 The CT scan of the cervical cancer patient. 
A

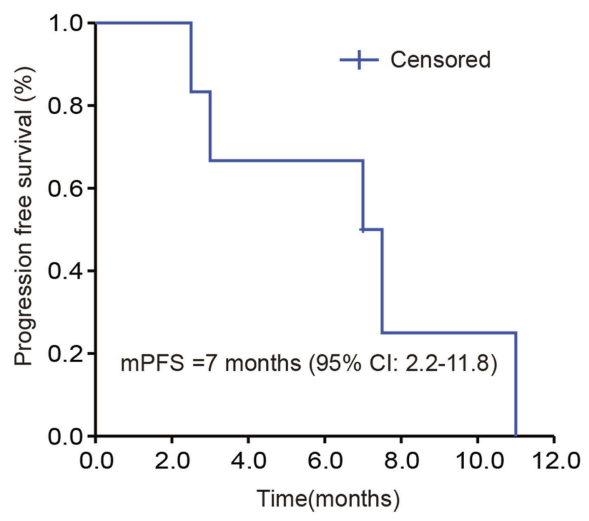

Figure 3 Survival curve of PFS and OS after apatinib treatment. (A. PFS; B. OS). Abbreviations: PFS, progression-free survival; OS, overall survival.

Table 3 Adverse events on treatment ( $n=6$ evaluable patients)

\begin{tabular}{|l|l|l|}
\hline Adverse event & Grade I/2 & Grade 3/4 \\
\hline Non-hematologic & & \\
Hypertension & $3(50 \%)$ & $\mathrm{I}(17 \%)$ \\
Mucositis & $2(33 \%)$ & \\
Fatigue & $\mathrm{I}(17 \%)$ & \\
Anorexia & $2(33 \%)$ & \\
Albuminuria & $\mathrm{I}(17 \%)$ & $\mathrm{I}(17 \%)$ \\
Hand-foot syndrome & $\mathrm{I}(17 \%)$ & \\
Gastrointestinal hemorrhage & $\mathrm{I}(17 \%)$ & \\
Hematologic & & $\mathrm{I}(17 \%)$ \\
Anemia & & \\
Leukopenia & $\mathrm{I}(17 \%)$ & $\mathrm{I}(17 \%)$ \\
Thrombocytopenia & & \\
\hline
\end{tabular}

activity of the extracellular regulated protein kinases signaling pathway. ${ }^{19}$ This property makes it is more possible to combine apatinib with convention chemotherapy.

Besides the efficacy of apatinib, we evaluated both clinic and laboratory side effects due to the retrospective nature of our study. In contrast to the previous experience from cytotoxic drugs, hematological toxicity is not that frequently, with one hematologic in $3 / 4$ grade, with one palette in $3 / 4$ grade, and with one leukocyte in $1 / 2$ grade. These may attribute to the nature molecule of apatinib which makes it not toxic in hematology. However, most patients reported some grade of non-hematological toxicity, while hypertension is the most common side effects with four patients and one of them suffered form 3/4 grade. Because of the antivascular effect, the microvessel was inhibited that cause the increased blood tension. Therefore, it is necessary to monitor the blood pressure when apatinib is given. Further, hand-foot syndrome is a common side effect of TKIs, although only a

\section{B}

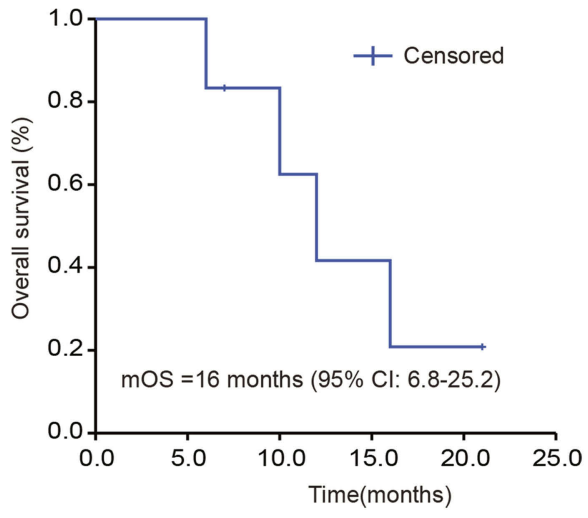

slight case was observed in this series, more attention should be paid. When this happens, supplementary B family vitamin and skin care would be helpful to release this syndrome. These profiles of complications were comparable with these of previously reported well-controlled trials using some dosage of apatinib in other malignancies. Summarily, most side effects were manageable by symptomatic treatment or dose modifications. However, it is worthy to notice that, one patient suffered the grade 3/4 hypertension and 3/4 palettes. But after reduction of usage dose, these side effects were fully released, no persistent toxicity was left. This may due to the individual response to the drug.

When compared with the conventional cytotoxic drugs such as vinorelbine or topotecan that are broadly used in the second-line setting, the effectiveness and side effects of apatinib are better or comparable. ${ }^{19}$ An OS of 17.6 months was observed with paclitaxel and platinum chemotherapy combined with bevacizumab, whereas an OS of 12.7 was observed when no bevacizumab was given. These OS are a bit longer than apatinib. However, the patients in GOG240 were native to platinum and paclitaxel chemotherapy. ${ }^{20}$ All the patients receiving apatinib had accepted at least paclitaxel and platinum-containing chemotherapy. It would be intriguing to see the outcome of the primary patients responses to the combination of apatinib and chemotherapy.

The side effects were observed with bevacizumab in that trial, such as gastrointestinal and genitourinary fistula which is a significant concern for both patients and physicians. ${ }^{21}$ Apatinib might be useful to as a compensation instead of bevacizumab for some patients in high risk for fistula such as these patients with radiotherapy in pelvic. Apart from efficacy and side effects, financial condition is another 


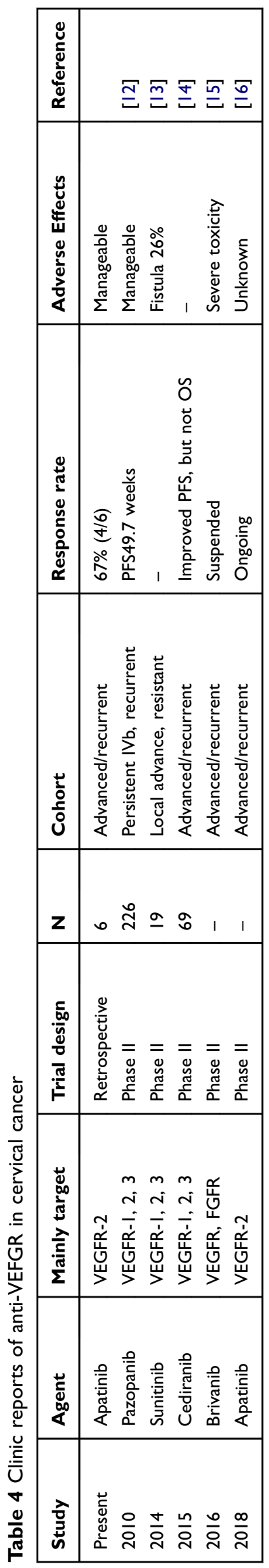

important factor in some condition, particularly in developing countries. Although the bevacizumab was approved in as the first line for cervical cancer based on the results of GOG240, this is currently only affordable in developed countries. It is estimated that bevacizumab added $\$ 73.791$ per 3.5 months of life gained ( $\$ 20,083$ per month of added life). ${ }^{22}$ This is overhigh in developing country, such as in China in which people with $\$ 1000$ per month averagely. ${ }^{23}$ Especially for the population of advanced cervical cancer patients who is not affordable for regular assessment of the cervical cancer screening program. Compared to the high cost of bevacizumab, apatinib is an optional compensation with the price around $\$ 300$ per month, which is still expensive but is more reachable for patients in China, or low-resource region.

In addition to apatinib and bevacizumab, a few other angiogenesis-targeting agents also have been reported in the treatment of cervical cancer. After literature retrieval, these reports are summarized in Table 4. Unfortunately, it seems patients benefit from TKIs but hamper by increased side effects. For example, pazopanib and cediranib might increase the outcomes. However, further investigation is still needed. ${ }^{24}$ In contrast to the manageable side effects in the current report, the toxicity happens in some drugs, for example, the usage of sunitinib and brivanib is hampered by the severe toxicity. ${ }^{15}$ This may attribute to the unspecific inhibition against VEGFRs the central events in angiogenesis. ${ }^{3}$ Moreover, the combination of bevazimnab and apatinib also seems to have synergistic effects in other solid tumors. ${ }^{25}$ A cocktail strategy aiming at increasing efficacies and reduction of side effects deserves further investigated. Considering the side effects, this searching for higher efficacy should be very careful to avoid the compensation in quality of life.

When interpreting the findings of this report, some limitations need to be kept in mind. Firstly, as a retrospective analysis, it is subjected to potential selection biases. Second, the small sample size precluded definitive conclusions. However, we conclude that apatinib is potentially effective and with manageable side effects in patients with heavily treated cervical cancer. Therefore, it is a possible treatment for patients as a second-line option. For a further perspective, a prospective, well designed, and controlled trial for the use of apatinib in cervical cancer patients is needed and worthy.

\section{Author contributions}

All authors contributed to data analysis, drafting or revising the article, gave final approval of the version to be published, and agree to be accountable for all aspects of the work. 


\section{Disclosure}

The authors have declared no conflicts of interest in this work.

\section{References}

1. Bray F, Ferlay J, Soerjomataram I, Siegel RL, Torre LA, Jemal A. Global cancer statistics 2018: GLOBOCAN estimates of incidence and mortality worldwide for 36 cancers in 185 countries. $C A$ Cancer J Clin. 2018;68(6):394-424. doi:10.3322/caac.21492

2. Torre LA, Bray F, Siegel RL, Ferlay J, Lortet-Tieulent J, Jemal A. Global cancer statistics, 2012. CA Cancer J Clin. 2015;65(2):87-108. doi: $10.3322 /$ caac. 21262

3. Crafton SM, Salani R. Beyond chemotherapy: an overview and review of targeted therapy in cervical cancer. Clin Ther. 2016;38 (3):449-458. doi:10.1016/j.clinthera.2016.02.007

4. Lee SH, Jeong D, Han Y-S, Baek MJ. Pivotal role of vascular endothelial growth factor pathway in tumor angiogenesis. Ann Surg Treat Res. 2015;89(1):1-8. doi:10.4174/astr.2015.89.1.1

5. Sawano A, Takahashi T, Yamaguchi S, Aonuma M, Shibuya M. Flt-1 but not KDR/Flk-1 tyrosine kinase is a receptor for placenta growth factor, which is related to vascular endothelial growth factor. Cell Growth Differ. 1996;7(2):213-221.

6. Stafl A, Mattingly RF. Angiogenesis of cervical neoplasia. Am J Obstet Gynecol. 1975;121(6):845-852.

7. Obermair A, Wanner C, Bilgi S, et al. Tumor angiogenesis in stage IB cervical cancer: correlation of microvessel density with survival. $\mathrm{Am}$ J Obstet Gynecol. 1998;178(2):314-319. doi:10.1016/s0002-9378 (98)80018-5

8. Tewari KS, Sill MW, Long HJ 3rd, et al. Improved survival with bevacizumab in advanced cervical cancer. $N$ Engl J Med. 2014;370 (8):734-743. doi:10.1056/NEJMoa1309748

9. Minion LE, Tewari KS. Cervical cancer - state of the science: from angiogenesis blockade to checkpoint inhibition. Gynecol Oncol. 2018;148(3):609-621. doi:10.1016/j.ygyno.2018.01.009

10. Zhao D, Hou H, Zhang X. Progress in the treatment of solid tumors with apatinib: a systematic review. Onco Targets Ther. 2018;11:4137-4147. doi:10.2147/OTT.S172305

11. Qiu H, Li J, Liu Q, Tang M, Wang Y. Apatinib, a novel tyrosine kinase inhibitor, suppresses tumor growth in cervical cancer and synergizes with Paclitaxel. Cell Cycle. 2018;17(10):1235-1244. doi:10.1080/15384101.2018.1471315

12. Shibuya M. Vascular endothelial growth factor (VEGF) and its receptor (VEGFR) signaling in angiogenesis: a crucial target for anti- and pro-angiogenic therapies. Genes Cancer. 2011;2(12):10971105. doi:10.1177/1947601911423031
13. Jo JC, Hong YS, Kim KP, et al. A prospective multicenter phase II study of sunitinib in patients with advanced aggressive fibromatosis. Invest New Drugs. 2014;32(2):369-376. doi:10.1007/s10637-0130059-0

14. Bender D, Sill MW, Lankes HA, et al. A phase II evaluation of cediranib in the treatment of recurrent or persistent endometrial cancer: an NRG Oncology/Gynecologic Oncology Group study. Gynecol Oncol. 2015;138(3):507-512.

15. Chan JK, Deng W, Higgins RV, et al. A phase II evaluation of brivanib in the treatment of persistent or recurrent carcinoma of the cervix: an NRG Oncology/Gynecologic Oncology Group study. Gynecol Oncol. 2017;146(3):554-559.

16. Zhou JG, Zhou NJ, Zhang Q, Feng YY, Zhou H. Apatinib for patients with advanced or recurrent cervical cancer: study protocol for an open-label randomized controlled trial. Trials. 2018;19 (1):500.

17. Adamska A, Falasca M. ATP-binding cassette transporters in progression and clinical outcome of pancreatic cancer: what is the way forward. World J Gastroenterol. 2018;24(29):3222-3238.

18. Hou XF, Xu LP, Song HY, et al. ECRG2 enhances the anti-cancer effects of cisplatin in cisplatin-resistant esophageal cancer cells via upregulation of p53 and downregulation of PCNA. World $J$ Gastroenterol. 2017;23(10):1796-1803.

19. Liu ZL, Jin BJ, Cheng CG, et al. Apatinib resensitizes cisplatinresistant non-small cell lung carcinoma A549 cell through reversing multidrug resistance and suppressing ERK signaling pathway. Eur Rev Med Pharmacol Sci. 2017;21(23):5370-5377.

20. Tewari KS, Sill MW, Long HJ 3rd, et al.Improved survival with bevacizumab in advanced cervical cancer. $N$ Engl J Med. 2014,370 (8):734-743

21. Tewari KS, Sill MW, Penson RT, et al. Bevacizumab for advanced cervical cancer: final overall survival and adverse event analysis of a randomised, controlled, open-label, phase 3 trial (Gynecologic Oncology Group 240). Lancet. 2017;390 (10103):1654-1663.

22. Minion LE, Bai J, Monk BJ, et al. A Markov model to evaluate costeffectiveness of antiangiogenesis therapy using bevacizumab in advanced cervical cancer. Gynecol Oncol. 2015;137(3):490-496.

23. William Carpenter J. Living in China on $\$ 1,000$ a month. Avaialble from: https://www.investopedia.com/articles/personalfinance/011416/living-china-1000-month.asp. Accessed October $11,2018$.

24. Alldredge JK, Tewari KS. Clinical trials of antiangiogenesis therapy in recurrent/persistent and metastatic cervical cancer. Oncologist. 2016;21(5):576-585

25. Rugo HS, Chien AJ, Franco SX, et al. A phase II study of lapatinib and bevacizumab as treatment for HER2-overexpressing metastatic breast cancer. Breast Cancer Res Treat. 2012;134(1):13-20.
OncoTargets and Therapy

\section{Publish your work in this journal}

OncoTargets and Therapy is an international, peer-reviewed, open access journal focusing on the pathological basis of all cancers, potential targets for therapy and treatment protocols employed to improve the management of cancer patients. The journal also focuses on the impact of management programs and new therapeutic agents and protocols on patient perspectives such as quality of life, adherence and satisfaction. The manuscript management system is completely online and includes a very quick and fair peer-review system, which is all easy to use. Visit http://www.dovepress.com/ testimonials.php to read real quotes from published authors. 